\title{
Economic modeling using evolutionary algorithms: the effect of a binary encoding of strategies
}

\author{
Ludo Waltman • Nees Jan van Eck • \\ Rommert Dekker • Uzay Kaymak
}

Published online: 9 June 2010

(C) The Author(s) 2010. This article is published with open access at Springerlink.com

\begin{abstract}
We are concerned with evolutionary algorithms that are employed for economic modeling purposes. We focus in particular on evolutionary algorithms that use a binary encoding of strategies. These algorithms, commonly referred to as genetic algorithms, are popular in agent-based computational economics research. In many studies, however, there is no clear reason for the use of a binary encoding of strategies. We therefore examine to what extent the use of such an encoding may influence the results produced by an evolutionary algorithm. It turns out that the use of a binary encoding can have quite significant effects. Since these effects do not have a meaningful economic interpretation, they should be regarded as artifacts. Our findings indicate that in general the use of a binary encoding is undesirable. They also highlight the importance of employing evolutionary algorithms with a sensible economic interpretation.
\end{abstract}

L. Waltman (凶) · N. J. van Eck · R. Dekker · U. Kaymak

Econometric Institute, Erasmus School of Economics,

Erasmus University Rotterdam, P.O. Box 1738,

3000 DR, Rotterdam, The Netherlands

e-mail: waltmanlr@cwts.leidenuniv.nl

N. J. van Eck

e-mail: ecknjpvan@cwts.leidenuniv.nl

R. Dekker

e-mail: rdekker@ese.eur.nl

U. Kaymak

e-mail:kaymak@ese.eur.nl 
Keywords Agent-based computational economics • Evolutionary algorithm • Genetic algorithm $\cdot$ Binary encoding $\cdot$ Premature convergence

JEL Classification $\mathrm{C} 63 \cdot \mathrm{C} 73 \cdot \mathrm{D} 43 \cdot \mathrm{D} 83$

\section{Introduction}

Evolutionary algorithms (EAs) are algorithms that are inspired by the process of natural evolution. EAs have their origins in the field of computer science, where they are mainly applied for optimization purposes. Nowadays, EAs are also regularly employed in the field of economics. In economic research, EAs frequently serve as a tool for modeling boundedly rational behavior. When EAs are applied as a modeling tool in economic research, a binary encoding of strategies is typically used. This means that strategies are represented by bit strings (i.e., strings of zeros and ones, often referred to as chromosomes) and that evolutionarily inspired operations such as crossover and mutation take place at the level of individual bits. EAs that use a binary encoding of strategies are commonly referred to as genetic algorithms. For early research in which genetic algorithms are employed, we refer to Miller $(1986,1996)$, Axelrod (1987), Marks (1992), Arifovic (1994, 1996), Andreoni and Miller (1995), and Dawid (1996). Examples of more recent research can be found in the work of, among others, Lux and Schornstein (2005), Alkemade et al. (2006, 2007, 2009), Arifovic and Maschek (2006), Wheeler et al. (2006), Xu (2006), Casari (2008), and Maschek (2010).

Researchers who apply genetic algorithms as a tool for modeling boundedly rational behavior typically do not justify why they use a binary encoding of strategies. If the agents whose behavior is being modeled have to make decisions that are intrinsically binary, such as decisions between cooperation and defection in a prisoner's dilemma (e.g., Axelrod 1987), the use of a binary encoding of strategies is a very natural choice. However, in the case of nonbinary decisions, such as decisions by firms on their production level (e.g., Arifovic 1994; Price 1997; Dawid and Kopel 1998; Franke 1998; Vriend 2000; Alkemade et al. 2006, 2007, 2009; Arifovic and Maschek 2006; Wheeler et al. 2006; Casari 2008; Maschek 2010), there is no clear reason for the use of a binary encoding of strategies.

In this paper, we examine to what extent the use of a binary encoding of strategies may influence the results of studies in which EAs are employed. It turns out that the use of a binary encoding can have quite significant effects. In general, these effects do not have a meaningful economic interpretation and should be regarded as artifacts. In order to avoid these artifacts, we argue that in most cases researchers should not use a binary encoding of strategies.

Our research is inspired by results reported by Alkemade et al. (2006, 2007, 2009; see also Waltman and Van Eck 2009b). Alkemade et al. show that under certain conditions an EA that is employed for modeling purposes may exhibit premature convergence. By premature convergence Alkemade et al. mean that 
different runs of the EA can lead to very different results. Alkemade et al. argue that premature convergence is caused by a too small population size. In this paper, we report results that point in a different direction. We show that the observation of premature convergence by Alkemade et al. depends crucially on their use of a binary encoding of strategies. Using the same economic environment as Alkemade et al. (i.e., a Cournot oligopoly market), we demonstrate that premature convergence does occur in the case of EAs with a binary encoding while it does not occur in the case of EAs without a binary encoding.

We note that the consequences of the use of a binary encoding of strategies are also studied extensively by Dawid (1996; see also Dawid and Kopel 1998). However, the approach taken by Dawid is quite different from the approach that we take in the present paper. Dawid focuses on EAs with a large population size, and he is concerned with aggregate results, that is, results averaged over many EA runs. We do not assume the population size to be large, and we are specifically interested in comparing results of individual EA runs. Another difference is that the crossover operator plays a crucial role in Dawid's approach while in our approach the crossover operator is not important at all.

The remainder of this paper is organized as follows. In Section 2, we introduce the Cournot market that we consider in this paper. In Sections 3 and 4, we present the various EAs that we study and we discuss the economic interpretation of EAs. We report the results of the computer simulations that we have performed in Section 5. Based on these results, we provide an elaborate analysis of the effect of a binary encoding of strategies in Section 6. Finally, in Section 7, we discuss the conclusions of our research.

\section{Cournot oligopoly market}

To analyze the effect of a binary encoding of strategies, we study the behavior of firms in a Cournot oligopoly market. To facilitate comparison, we consider exactly the same Cournot market as Alkemade et al. (2006, 2007, 2009). For other studies in which quantity competition among firms is modeled using EAs, we refer to Arifovic (1994), Price (1997), Dawid and Kopel (1998), Franke (1998), Vriend (2000), Arifovic and Maschek (2006), Wheeler et al. (2006), Casari (2008), and Maschek (2010).

The Cournot market that we consider has the following characteristics: The number of firms equals four, firms produce perfect substitutes, the demand function is linear, firms have identical cost functions, and marginal cost is constant. The inverse demand function is given by:

$$
p=\max \left(256-\sum_{i=1}^{4} q_{i}, 0\right)
$$


where $p$ denotes the market price and $q_{i}$ denotes firm $i$ 's production level. Firm $i$ 's total cost equals $c_{i}=56 q_{i}$. Hence, it follows that firm $i$ 's profit is given by:

$$
\pi_{i}=p q_{i}-c_{i}=q_{i} \max \left(200-\sum_{i^{\prime}=1}^{4} q_{i^{\prime}},-56\right) .
$$

A Nash (or Cournot) equilibrium is obtained if each firm chooses a production level that maximizes its profit given the production levels of its competitors. This means that in a Nash equilibrium $\partial \pi_{i} / \partial q_{i}=0$ for $i=1, \ldots, 4$. It is easy to see that the Cournot market that we consider has a Nash equilibrium in which each firm produces a quantity of 40 . Each firm makes a profit of 1600 in the Nash equilibrium. In addition to a Nash equilibrium, the Cournot market that we consider also has a competitive (or Walrasian) equilibrium. This equilibrium is obtained if firms are not aware of their influence on the market price and therefore behave as price takers. In the competitive equilibrium, the four firms jointly produce a quantity of 200 and each firm makes a profit of 0 .

\section{Evolutionary algorithms}

As discussed by Vriend (2000), there are two quite different ways in which EAs can be employed to model the behavior of economic agents. In the individual learning approach, each agent learns exclusively from its own experience (e.g., Arifovic 1994; Price 1997; Arifovic and Maschek 2006; Casari 2008). This is modeled through the use of a separate EA for each agent. In the social learning approach, each agent learns not only from its own experience but also from the experience of other agents (e.g., Arifovic 1994; Dawid and Kopel 1998; Franke 1998; Alkemade et al. 2006, 2007, 2009). This is modeled through the use of a single EA for all agents together. The social learning approach seems to be more popular than the individual learning approach (Arifovic and Maschek 2006). In this paper, we focus on the social learning approach.

An important observation about the social learning approach is made by Alkemade et al. $(2006,2007,2009)$. They note that the social learning approach can be implemented in two quite different ways. On the one hand, one can employ an EA with a population size that equals the number of interacting agents (e.g., Arifovic 1994; Dawid and Kopel 1998; Franke 1998; Vriend 2000). This results in a one-to-one relationship between strategies and agents. In the case of the Cournot oligopoly market discussed in the previous section, the EA would have a population size of four (since there are four firms in the market). On the other hand, one can employ an EA with a population size that exceeds the number of interacting agents (e.g., Axelrod 1987; Andreoni and Miller 1995; Dawid 1996, Sections 4.5 and 5.3; Miller 1996). Strategies are then evaluated using some matching mechanism. In the case of the Cournot oligopoly market discussed in the previous section, the EA would have a population size greater than four. Alkemade et al. show that the two ways in which the social learning approach can be implemented can lead to very 




Fig. 1 General form of the six EAs considered in this paper

different results. ${ }^{1}$ In this paper, we only employ EAs with a population size that equals the number of interacting agents. We take this approach because it is very suitable for demonstrating how the use of a binary encoding of strategies can lead to artifacts.

We consider six different EAs in this paper. We refer to these EAs as EA1 to EA6. Each of the EAs provides a slightly different model of the behavior of firms in the Cournot market discussed in the previous section. The six EAs all have the same general form. This general form is shown in Fig. 1. Each EA works on a population of four strategies. A strategy corresponds with the production level of one of the four firms in the Cournot market. The EAs all impose the constraint that the production level of a firm must lie between 0 and 127, and they all randomly generate an initial population by drawing four strategies from a uniform distribution over all possible strategies. In each iteration of an EA, the profit resulting from each of the four strategies in the current population is calculated using Eq. 2. Based on the profits of the four strategies, the fitness values of the strategies are calculated according to:

$$
f_{i}=\max \left(\frac{\pi_{i}-\mu}{\sigma}+2,0\right),
$$

where $\mu$ and $\sigma$ denote, respectively, the mean and the standard deviation of the profits of the strategies, that is:

$$
\begin{gathered}
\mu=\frac{\sum_{i=1}^{4} \pi_{i}}{4}, \\
\sigma=\sqrt{\frac{\sum_{i=1}^{4}\left(\pi_{i}-\mu\right)^{2}}{4} .}
\end{gathered}
$$

The above transformation from profits to fitness values is sometimes referred to as sigma scaling (e.g., Mitchell 1996) or sigma truncation (e.g., Goldberg

\footnotetext{
${ }^{1}$ In the evolutionary game theory literature, similar observations have been made by various researchers, for example by Hansen and Samuelson (1988) and Rhode and Stegeman (1996) in an economic context and by Schaffer (1988) in a biological context.
} 
1989). The transformation is used by, for example, Axelrod (1987), Andreoni and Miller (1995), Miller (1996), and Franke (1998). The six EAs that we study all use roulette wheel selection (e.g., Goldberg 1989; Mitchell 1996), also known as fitness-proportionate selection. This means that in each iteration of an EA the selection operator generates a new population of strategies by randomly drawing four strategies from the old population. Strategies are drawn independently and with replacement. The probability that a strategy is drawn is proportional to the fitness of the strategy given by Eq. 3 .

The six EAs that we consider differ from each other on the following four dimensions:

1. The type of strategy that is used, that is, integer production levels or realvalued production levels.

2. Whether a binary encoding of strategies is used or not.

3. Whether a crossover operator is used or not.

4. The type of mutation operator that is used.

The differences between the EAs are summarized in Table 1. We now discuss the specific characteristics of each of the EAs.

\subsection{EA1}

EA1 is a standard genetic algorithm (e.g., Goldberg 1989; Mitchell 1996). It uses integer production levels and a binary encoding of strategies. A strategy is represented by a bit string of length seven. The production level corresponding to a bit string $\left(b_{1}, \ldots, b_{7}\right)$, where $b_{j} \in\{0,1\}$ denotes the value of the $j$ th bit in the string, is given by:

$$
q=\sum_{j=1}^{7} 2^{7-j} b_{j} .
$$

EA1 uses a single-point crossover operator (e.g., Mitchell 1996) with a crossover rate of 1 . The mutation operator used by EA1 randomly determines for each bit in a bit string whether to invert the bit or not. The mutation rate equals 0.001 , which means that each bit has a probability of 0.001 of being inverted. We note that EA1 is very similar to the EA employed by Alkemade et al. $(2006,2007,2009)$.

Table 1 Overview of the differences between the six EAs considered in this paper

\begin{tabular}{lllllll}
\hline & EA1 & EA2 & EA3 & EA4 & EA5 & EA6 \\
\hline Strategy & Integer & Integer & Integer & Integer & Real number & Integer \\
Binary encoding & Yes & Yes & No & No & No & Yes (Gray) \\
Crossover & Yes & No & No & No & No & Yes \\
Mutation & Bit flip & Bit flip & Unif. dist. & \pm 1 & Norm. dist. & Bit flip \\
\hline
\end{tabular}




\subsection{EA2}

EA2 is identical to EA1 except that it does not use a crossover operator.

\subsection{EA3}

Like EA2, EA3 uses integer production levels and does not use a crossover operator. Unlike EA2, EA3 does not use a binary encoding of strategies. EA3 also uses a different mutation operator than EA2. The probability that a strategy is being mutated equals 0.01 . If a strategy is being mutated, it is replaced by a random new strategy that is drawn from a uniform distribution over all possible strategies. In the economic literature, EAs similar to EA3 are employed by Ünver (2001), Dawid and Dermietzel (2006), and Haruvy et al. (2006).

\subsection{EA4}

EA4 is identical to EA3 except that it uses a different mutation operator. The probability that a strategy is being mutated equals 0.01 . If a strategy is being mutated, the corresponding production level is either increased by one or decreased by one (both with a probability of 0.5 ). The increase or decrease does not take place if the resulting new production level would be below 0 or above 127.

\subsection{EA5}

Like EA3 and EA4, EA5 does not use a binary encoding of strategies and also does not use a crossover operator. Unlike EA3 and EA4, EA5 uses real-valued production levels. EA5 also uses a different mutation operator than EA3 and EA4. The probability that a strategy is being mutated equals 0.01 . If a strategy is being mutated, the corresponding production level is updated according to:

$$
q_{\text {new }}=\min \left(\max \left(q_{\text {old }}+N\left(0, s^{2}\right), 0\right), 127\right),
$$

where $q_{\text {old }}$ denotes the production level before mutation, $q_{\text {new }}$ denotes the production level after mutation, and $N\left(0, s^{2}\right)$ denotes a normally distributed random variable with mean 0 and standard deviation $s$. EA5 uses a value of 1 for the parameter $s$. We note that EA5 is somewhat similar to what is referred to as an evolution strategy in the computer science literature (e.g., Beyer 2001; Beyer and Schwefel 2002). In the economic literature, EAs similar to EA5 are employed by Sellgren (2001), Gerding et al. (2003), Lux and Schornstein (2005), and Clemens and Riechmann (2006).

\subsection{EA6}

EA6 is identical to EA1 except that it does not use an ordinary binary encoding of strategies. Instead, it uses a so-called Gray coding of strategies. Like in 
Table 2 Illustration of the Gray coding used in EA6

\begin{tabular}{ll}
\hline Production level & Bit string \\
\hline 0 & 0000000 \\
1 & 0000001 \\
2 & 0000011 \\
3 & 0000010 \\
4 & 0000110 \\
$\ldots$ & $\ldots$ \\
126 & 1000001 \\
127 & 1000000 \\
\hline
\end{tabular}

EA1, strategies are represented by bit strings of length seven. However, the transformation from bit strings to production levels is different from the transformation used in EA1, that is, it is different from Eq. 6. In EA6, the transformation from bit strings to production levels is performed in such a way that bit strings corresponding to consecutive production levels always differ by only one bit. This is referred to as a Gray coding of strategies. The Gray coding used in EA6 is illustrated in Table 2.

In the literature, Gray codings of strategies are used only rarely. Usually, an ordinary binary encoding of strategies is used, like in EA1 and EA2. Examples of the use of Gray codings of strategies are provided by Arifovic (1996) and Maschek (2010). Arifovic states that "the Gray coding ensures that, if a small number of bits within a binary string change, this will correspond to a small change in a decoded integer or real number" (p. 525). However, this is not correct. Even if a Gray coding of strategies is used, a change of a small number of bits in a bit string may still correspond to a large change in the decoded value. This can be seen in Table 2. The bit strings 0000000 and 1000000 differ by only one bit, but they correspond to two very different production levels, namely 0 and 127 , respectively.

\section{Economic interpretation of evolutionary algorithms}

In many papers in which EAs are applied as an economic modeling tool, relatively little attention is paid to the economic interpretation of EAs. ${ }^{2}$ In the present paper, the economic interpretation of EAs is a central issue and hence requires serious attention. In this section, we therefore summarize the various ways in which EAs are interpreted in the literature.

An EA works on a population of strategies. What exactly does a population of strategies represent? As discussed in the previous section, we need to make a distinction between the individual learning approach and the social learning

\footnotetext{
${ }^{2}$ A notable exception is a paper by Chattoe (1998), in which the economic interpretation of EAs is critically discussed.
} 
approach (Vriend 2000). ${ }^{3}$ In the individual learning approach, a separate EA is used for each agent. Hence, each agent has its own population of strategies. Arifovic (1994) interprets a population of strategies in the individual learning approach as "an agent's mutually competing ideas about what his behavior in a given environment should be" (p. 15). According to Dawid (1996), the interpretation of the individual learning approach has several weaknesses. For example, the individual learning approach assumes that an agent is able to determine the performance of a strategy without actually executing the strategy. This may be a strong assumption in many contexts. Price (1997), however, argues that in certain cases the assumption may be justified, in particular in the case of firms that perform scenario analysis. ${ }^{4}$ In the social learning approach, a single EA is used for all agents together. In this approach, the population of strategies can be interpreted in two ways (Alkemade et al. $2006,2007,2009$ ). In one interpretation, the population size equals the number of interacting agents and strategies and agents are related in a one-to-one manner. Each strategy then simply represents the strategy of one particular agent. This is the interpretation that we follow in this paper. In the other interpretation, the population size exceeds the number of interacting agents. The population of strategies can then be seen as a pool of strategies that are commonly known to all agents. When interacting with each other, agents randomly choose a strategy from the strategy pool.

In most EAs that are employed for economic modeling, a binary encoding of strategies is used. In general, it is unclear how the use of such an encoding can be given a sensible economic interpretation. Most researchers ignore this issue. An exception is Brenner (2006), who points out that the use of a binary encoding of strategies may lead to difficulties with the interpretation of the crossover operator. A somewhat similar comment is made by Dawid (1996).

We now discuss the economic interpretation of the selection, crossover, and mutation operators of an EA. Our focus is on the social learning approach.

Following Chattoe (1998), we distinguish between two interpretations of the selection operator. The first interpretation can be used only if there is a one-to-one relationship between strategies and agents. According to this interpretation, the selection operator models the removal of unsuccessful agents from the economic environment. An example is the removal of unprofitable firms from the market due to bankruptcy. The second interpretation, which is used by most researchers, states that the selection operator models the imitation of successful strategies. According to this interpretation, agents have information on the past performance of strategies and tend to imitate those strategies that were most successful in the past. The details of this

\footnotetext{
${ }^{3}$ Arifovic (1994) refers to these approaches as the multiple-population design and the singlepopulation design. Chattoe (1998) refers to the approaches as the mental interpretation and the population interpretation of an EA.

${ }^{4} \mathrm{An}$ alternative assumption is that agents try out the various strategies they have in mind and that they update their strategies only after they have obtained a sufficient amount of information on each strategy's performance. An assumption like this is made by Vriend (2000).
} 
interpretation depend on the type of selection operator that is used. For example, roulette wheel or fitness-proportionate selection, which is the most commonly used selection operator, assumes that an agent has information on the past performance of all strategies. On the other hand, tournament selection, which is used in some papers (e.g., Bullard and Duffy 1998; Van Bragt et al. 2001; Dawid and Dermietzel 2006), assumes that an agent has information on the past performance of only a limited number of strategies. Another thing to realize is that most selection operators assume that agents update their strategies simultaneously rather than one by one. The assumption of simultaneous updating of strategies may not always be realistic. A comparison of simultaneous and non-simultaneous updating of strategies is performed by Dawid and Dermietzel (2006). They find that the two strategy updating regimes may lead to significantly different results. We note that the issue of the appropriate strategy updating regime has also received considerable attention in the biological literature (e.g., Huberman and Glance 1993).

The economic interpretation of the crossover and mutation operators is quite straightforward. The crossover operator is typically interpreted as the exchange of ideas or information. Hence, the crossover operator models communication between agents (or industrial espionage, as suggested by Dawid and Kopel 1998). The mutation operator is usually interpreted as the effect of innovation. Innovation may be due to deliberate experimentation or unintended errors.

\section{Simulation results}

In this section, we report the results of the computer simulations that we have performed. ${ }^{5}$ Each of the six EAs discussed in Section 3 was run 100 times, each time using different random numbers. Each run lasted 10,000 iterations. The results reported below are fairly robust to changes in the values of the various EA parameters. By changing parameter values, somewhat different results may be obtained, but the analysis will remain essentially unchanged. The results reported below are also robust to changes in the transformation from profits to fitness values. We further experimented with simulation runs that lasted one million instead of 10,000 iterations, but this also did not affect the analysis in any fundamental way.

The results of six selected runs of EA1 are shown in Fig. 2. Each graph in the figure corresponds with one run of EA1. The graphs display how the average production level of the four firms in the market evolves over time. In the graphs in panels (a), (b), and (c), firms' average production level stabilizes fairly quickly, respectively at a quantity of 47,50 , and 64 . These results are quite typical. In almost all 100 runs of EA1, we observe that firms'

\footnotetext{
${ }^{5}$ The software used to obtain the results is available online at http://www.ludowaltman.nl/ binaryencoding/. The software runs in MATLAB.
} 
a

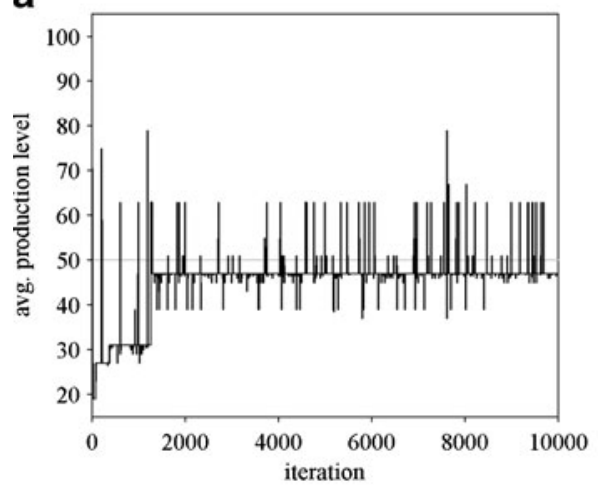

C

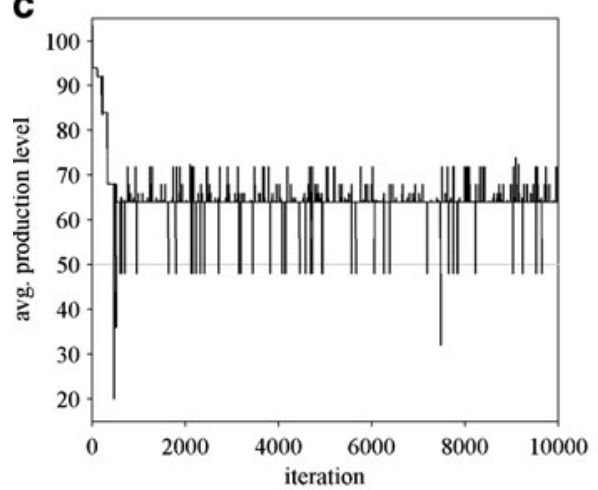

e

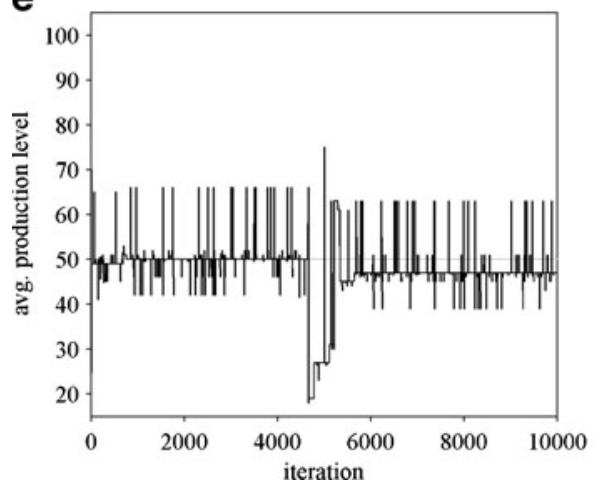

b

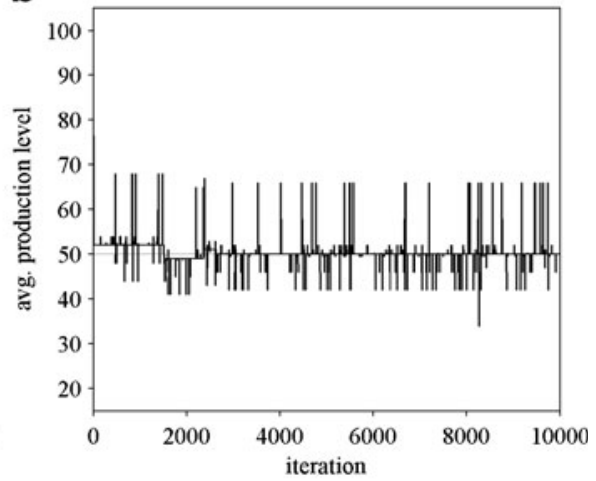

d

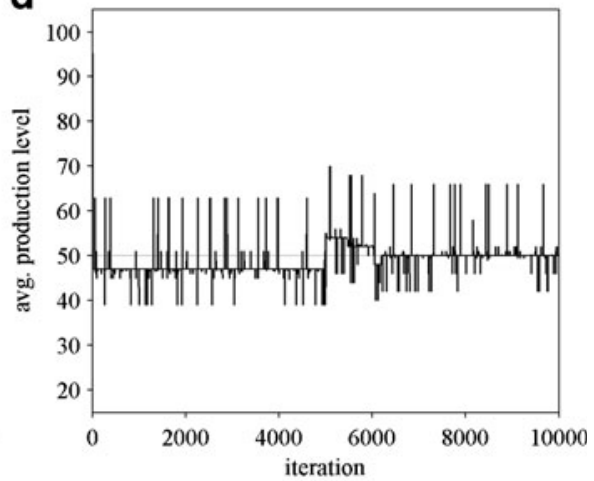

f

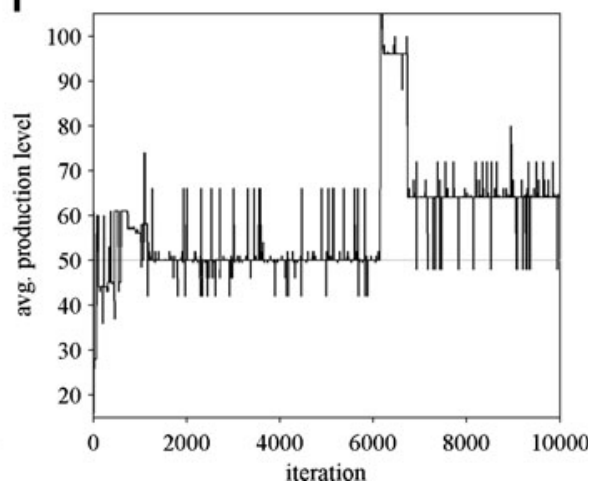

Fig. 2 Results of six selected runs of EA1. The graphs display for each run how firms' average production level evolves over time. The horizontal line in each graph indicates the competitive equilibrium quantity of 50

average production level stabilizes at one of these three quantities. However, firms' average production level does not always remain stabilized at the same quantity during an entire run. In a few runs of EA1, we find that firms' average production level switches from one stable quantity to another. Panels (d), (e), and (f) of Fig. 2 provide examples of such runs. 
In panel (a) of Fig. 3, the results of all 100 runs of EA1 are averaged. As can be seen, on average firms' production level stabilizes at a quantity of about 52. Based on the 100 runs of EA1, the distribution of firms' average production level at the end of a run can be determined. This distribution is displayed in panel (b) of Fig. 3. It turns out that in somewhat more than half of the runs firms' average production level after 10,000 iterations equals the stable quantity of 50. In most other runs, firms' average production level after 10,000 iterations equals either the stable quantity of 47 or the stable quantity of 64. There are a few runs in which firms' average production level after 10,000 iterations does not equal one of the three stable quantities. In most of these runs, this is probably due to small disturbances caused by the mutation operator.

The results that we have obtained using EA1 are very similar to the results reported by Alkemade et al. (2006, 2007, 2009). ${ }^{6}$ Like Alkemade et al., we find that in different EA runs firms' average production level stabilizes at different quantities. This phenomenon is referred to as premature convergence by Alkemade et al. We further find that on average firms' production level stabilizes at a quantity above 50 . This means that on average firms produce a larger quantity than in the competitive equilibrium of the Cournot oligopoly market (see Section 2). This finding is also in agreement with the results reported by Alkemade et al.

We now turn to EA2. The results obtained using EA2 are shown in panels (c) and (d) of Fig. 3. It is clear that the results of EA2 are quite similar to the results of EA1. Like EA1, EA2 leads to premature convergence. Firms' average production level again stabilizes at a quantity of 47,50 , or 64 . The similarity between the results of EA1 and EA2 is not surprising. The only difference between the two EAs is that EA1 uses a crossover operator while EA2 does not use such an operator. In an earlier paper (Waltman and Van Eck 2009a), we have shown mathematically that, if the mutation rate is small and some technical assumptions are satisfied, the effect of the use of a crossover operator on the results produced by an EA tends to be negligible in the long run. The results shown in Fig. 3 are in line with this theoretical finding.

Finally, we consider EA3, EA4, EA5, and EA6. The results obtained using these EAs are shown in panels (e) to (l) of Fig. 3. As can be seen, in the long run the four EAs produce quite similar results. The only noteworthy difference is that the results of EA3 are more volatile than the results of EA4, EA5, and EA6. However, this is to be expected, since EA3 uses a more disruptive mutation operator than the other three EAs (see Section 3). What is more interesting to look at is the difference between the results of EA3, EA4, EA5, and EA6 on the one hand and the results of EA1 and EA2 on the other hand.

\footnotetext{
${ }^{6}$ Due to an error in their computer simulations, the results reported by Alkemade et al. (2006, 2007) are not entirely correct. For a correction of the results, see Alkemade et al. (2009). For some additional comments on the results, see Waltman and Van Eck (2009b).
} 

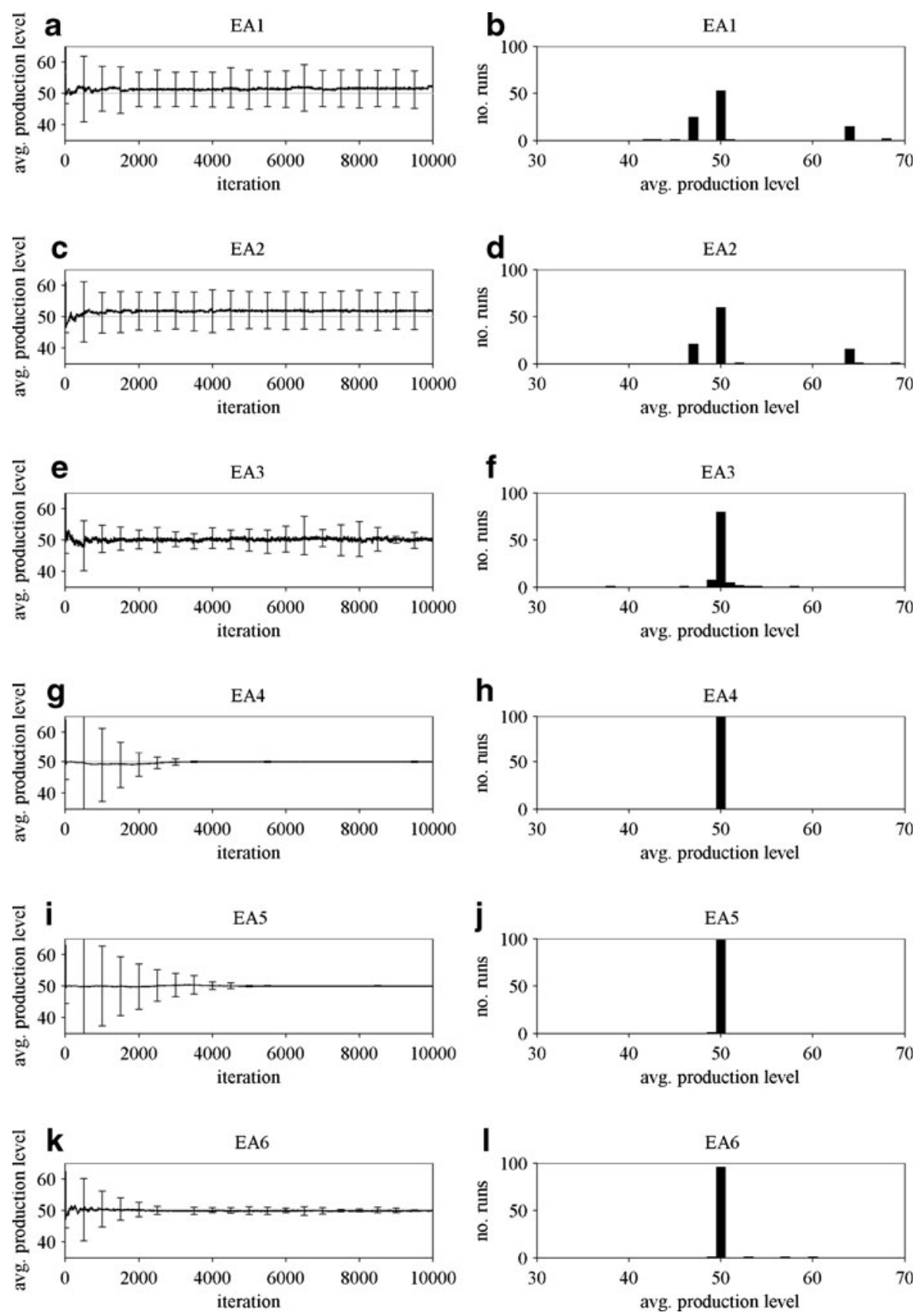

Fig. 3 Results of EA1 to EA6. The graphs in the left panels display for each EA how firms' average production level evolves over time. The results shown in the graphs are averages over 100 EA runs. Error bars indicate standard deviations. The horizontal line in each graph indicates the competitive equilibrium quantity of 50. The histograms in the right panels display for each EA the distribution of firms' average production level at the end of a run. The distributions are based on 100 EA runs 
When looking at the aggregate results of 100 EA runs (see the left panels of Fig. 3), it can be seen that firms' average production level stabilizes at a quantity of 50 in the case of EA3, EA4, EA5, and EA6 while it stabilizes at a quantity of about 52 in the case of EA1 and EA2. Hence, in the case of EA3, EA4, EA5, and EA6, firms on average produce the quantity associated with the competitive equilibrium of the Cournot market (see Section 2). In the case of EA1 and EA2, on the other hand, firms on average produce a quantity that is larger than the competitive equilibrium quantity. When looking at the results of individual EA runs (see the right panels of Fig. 3), it turns out that firms' average production level stabilizes around a quantity of 50 in the case of EA3, EA4, EA5, and EA6 while it stabilizes at a quantity of 47, 50, or 64 in the case of EA1 and EA2. Hence, premature convergence only takes place in the case of EA1 and EA2. It does not take place in the case of the other four EAs. Based on the above observations, it is clear that, both at the aggregate level and at the level of individual runs, EA3, EA4, EA5, and EA6 produce fundamentally different results than EA1 and EA2.

The principal difference between EA1 and EA2 on the one hand and EA3, EA4, and EA5 on the other hand is that EA1 and EA2 use a binary encoding of strategies while EA3, EA4, and EA5 do not use such an encoding (see Table 1). EA6 also uses a binary encoding of strategies, but this is a special type of binary encoding, namely a Gray coding. Based on the results reported in this section, it seems that the use of a binary encoding of strategies can have quite significant effects. In the next section, we provide an analysis of these effects, in particular of the phenomenon of premature convergence.

\section{Analysis of the effect of a binary encoding of strategies}

For each of the six EAs considered in the previous section, it turned out that in at least a substantial number of runs firms' average production level stabilized at a quantity of 50 , that is, at the quantity associated with the competitive equilibrium of the Cournot oligopoly market. There is a straightforward explanation for this finding. Suppose that in some iteration of an EA the population consists of four identical strategies, each corresponding with a production level of 50. The market price then equals firms' constant marginal cost, and each strategy therefore results in a profit of 0 . Suppose now that the mutation operator changes one of the strategies in the population. We refer to this strategy as strategy A. Strategy A's production level may either increase or decrease. If strategy A's production level increases, the total quantity produced in the market will increase and, as a consequence, the market price will fall below firms' constant marginal cost. All four strategies in the population will then result in a loss, but strategy A will result in a larger loss than the other strategies. If on the other hand strategy A's production level decreases, the total quantity produced in the market will decrease and, as a consequence, the market price will rise above firms' constant marginal cost. All four strategies in the population will then result in a profit, but strategy 
A will result in a smaller profit than the other strategies. Hence, regardless of whether strategy A's production level increases or decreases, the fitness of strategy A will always be lower than the fitness of the other strategies in the population. As a consequence, the probability that in the next iterations of the EA strategy A remains in the population is quite low. Most likely, within one or a few iterations, the selection operator will remove strategy A from the population. The population will then return to its original state, that is, it will again consist of four identical strategies, each corresponding with a production level of 50 .

The above mechanism explains why in the EAs considered in the previous section firms' average production level tends to stabilize at the competitive equilibrium quantity of 50. It should be noted that the mechanism has been discussed quite extensively in the evolutionary game theory literature. The mechanism was first discussed by Hansen and Samuelson (1988) and Schaffer (1989), and a comprehensive mathematical treatment of the mechanism was provided by Vega-Redondo (1997). A discussion of the mechanism can also be found in a study by Vriend (2000) on EA modeling in a Cournot oligopoly environment.

As discussed in the previous section, the fundamental difference between the results obtained using EA1 and EA2 on the one hand and the results obtained using EA3, EA4, EA5, and EA6 on the other hand is that in the case of EA1 and EA2 firms' average production level can stabilize not only at a quantity of 50 but also at a quantity of 47 or 64 . This phenomenon of multiple stable quantities is referred to as premature convergence by Alkemade et al. (2006, 2007, 2009). We now show that the premature convergence phenomenon is caused by the use of a binary encoding of strategies.

Suppose that in some iteration of EA1 or EA2 the population consists of four identical strategies, each corresponding with a production level of 47 . Each strategy is then represented by the bit string 0101111. Suppose now that out of the 28 bits used to represent the four strategies in the population exactly one bit is inverted by the mutation operator. This means that after applying the mutation operator one of the four strategies in the population has changed while the other three strategies have not changed. We refer to the strategy that has changed as strategy A. The first column of Table 3 lists seven bit strings. It is clear that one of these bit strings must represent strategy A

Table 3 Effect of the inversion of a single bit given a population in which each strategy corresponds with a production level of 47 (represented by the bit string 0101111)

\begin{tabular}{lccc}
\hline $\begin{array}{l}\text { Bit string } \\
\text { strategy A }\end{array}$ & $\begin{array}{l}\text { Production level } \\
\text { strategy A }\end{array}$ & $\begin{array}{l}\text { Profit } \\
\text { strategy A }\end{array}$ & $\begin{array}{l}\text { Profit other } \\
\text { strategies }\end{array}$ \\
\hline 0101110 & 46 & 598 & 611 \\
0101101 & 45 & 630 & 658 \\
0101011 & 43 & 688 & 752 \\
0100111 & 39 & 780 & 940 \\
0111111 & 63 & -252 & -188 \\
0001111 & 15 & 660 & 2,068 \\
1101111 & 111 & $-5,772$ & $-2,444$ \\
\hline
\end{tabular}


(which one depends on which bit has been inverted). For each bit string, the corresponding production level is listed in the second column of the table. The last two columns of the table list for each production level the resulting profit of strategy A as well as the resulting profit of the other three strategies in the population, that is, the strategies corresponding with a production level of 47. Profits were calculated using Eqs. 1 and 2. As can be seen in the table, the profit of strategy A will always be smaller than the profit of the other three strategies, regardless of which bit has been inverted. This means that within one or a few iterations of the EA the selection operator will most likely remove strategy A from the population. The population will then return to its original state, that is, it will again consist of four identical strategies, each corresponding with a production level of 47.

In the case of EA1 and EA2, the above mechanism shows that, if the population is in a state in which each strategy corresponds with a production level of 47, the inversion of a single bit is unlikely to upset this state for more than a few iterations. Of course, things may be different when the mutation operator inverts two or more bits at the same time. However, this happens only very rarely. (Given a mutation rate of 0.001 , this happens on average once in every 2692 iterations of an EA.) The above mechanism therefore explains why in the case of EA1 and EA2 firms' average production level can stabilize at a quantity of 47.

The same explanation also holds for a quantity of 64. This quantity corresponds with the bit string 1000000 . The bit strings that can be obtained by inverting a single bit are listed in the first column of Table 4. This table has a similar structure as Table 3. Like in Table 3, the profits listed in the third column of Table 4 are always smaller than those listed in the fourth column. This indicates that, if the population is in a state in which each strategy corresponds with a production level of 64, the inversion of a single bit is unlikely to upset this state for more than a few iterations. Taking into account that the simultaneous inversion of two or more bits happens only very rarely, this explains why 64 is a stable quantity in the case of EA1 and EA2.

A question that remains is whether in the case of EA1 and EA2 there are other stable quantities in addition to 47, 50, and 64. To answer this question, we calculated tables similar to Tables 3 and 4 for all integer quantities between 0 and 127 . It turned out that 47, 50, and 64 are the only quantities for which

Table 4 Effect of the inversion of a single bit given a population in which each strategy corresponds with a production level of 64 (represented by the bit string 1000000)

\begin{tabular}{llll}
\hline $\begin{array}{l}\text { Bit string } \\
\text { strategy A }\end{array}$ & $\begin{array}{l}\text { Production level } \\
\text { strategy A }\end{array}$ & $\begin{array}{l}\text { Profit } \\
\text { strategy A }\end{array}$ & $\begin{array}{l}\text { Profit other } \\
\text { strategies }\end{array}$ \\
\hline 1000001 & 65 & $-3,640$ & $-3,584$ \\
1000010 & 66 & $-3,696$ & $-3,584$ \\
1000100 & 68 & $-3,808$ & $-3,584$ \\
1001000 & 72 & $-4,032$ & $-3,584$ \\
1010000 & 80 & $-4,480$ & $-3,584$ \\
1100000 & 96 & $-5,376$ & $-3,584$ \\
0000000 & 0 & 0 & 512 \\
\hline
\end{tabular}


the inversion of a single bit always results in a smaller profit for the mutated strategy than for the three non-mutated strategies. 47, 50, and 64 are therefore the only stable quantities. All other quantities are unstable. Consider for example Table 5. This table was calculated for a quantity of 48. As can be seen in the table, the inversion of one of the three rightmost bits results in a larger profit for the mutated strategy (referred to as strategy A in the table) than for the three non-mutated strategies. This indicates that, given a population in which each strategy corresponds with a production level of 48 , the inversion of a single bit can relatively easily trigger a transition to a completely different population. This makes 48 an unstable quantity.

We have now shown how the use of a binary encoding of strategies causes the premature convergence observed in the case of EA1 and EA2. Based on our analysis, it is clear that the phenomenon of premature convergence depends crucially on the use of a binary encoding of strategies. An obvious question then is why no premature convergence is observed in the case of EA6. Like EA1 and EA2, EA6 uses a binary encoding of strategies. In the case of EA6, however, a special type of binary encoding is used, namely a Gray coding. Why is no premature convergence observed when a Gray coding is used? This can be explained as follows. Suppose that in some iteration of EA6 the population consists of four identical strategies. These strategies correspond with a production level of $q$, where $q$ denotes an integer below 50. Suppose further that the mutation operator inverts a single bit. We refer to the strategy that has changed as strategy A. Due to the use of a Gray coding of strategies, it is always possible that the inversion of a single bit causes one of the four production levels to increase by one. (Notice that this is not the case when an ordinary binary encoding of strategies is used.) Suppose that the production level corresponding with strategy $\mathrm{A}$ has indeed increased by one, from $q$ to $q+1$. It is clear that strategy A then results in a larger profit than the other three strategies in the population. This means that, due to the effect of the selection operator, it is quite likely that strategy A will spread through the population. As a consequence, within a few iterations, all four strategies in the population may correspond with a production level of $q+1$. This mechanism explains why any quantity below 50 is unstable in the case of EA6. A similar mechanism explains why any quantity above 50 is unstable. Hence, unlike in the case of EA1 and EA2, 50 is the only stable quantity in the case of EA6. Because of this, EA6 does not exhibit premature convergence.

Table 5 Effect of the inversion of a single bit given a population in which each strategy corresponds with a production level of 48 (represented by the bit string 0110000)

\begin{tabular}{lccc}
\hline $\begin{array}{l}\text { Bit string } \\
\text { strategy A }\end{array}$ & $\begin{array}{l}\text { Production level } \\
\text { strategy A }\end{array}$ & $\begin{array}{l}\text { Profit } \\
\text { strategy A }\end{array}$ & $\begin{array}{l}\text { Profit other } \\
\text { strategies }\end{array}$ \\
\hline 0110001 & 49 & 343 & 336 \\
0110010 & 50 & 300 & 288 \\
0110100 & 52 & 208 & 192 \\
0111000 & 56 & 0 & 0 \\
0100000 & 32 & 768 & 1,152 \\
0010000 & 16 & 640 & 1,920 \\
1110000 & 112 & $-6,272$ & $-2,688$ \\
\hline
\end{tabular}




\section{Conclusions}

In a paper on EA modeling, Dawid and Kopel (1998) warn that "we have to be aware of the fact that simulation results may crucially depend on implementation details which have hardly any economic meaning" (p. 311). The present paper can be seen as an illustration of this important but somewhat overlooked point. In the context of quantity competition among firms, it is difficult if not impossible to give a sensible economic interpretation to the use of a binary encoding of strategies. In fact, the use of a binary encoding seems merely a relic from the genetic algorithm literature in the field of computer science. Of course, nothing would be wrong with the use of a binary encoding if its effect on the results produced by an EA were insignificant. However, our computer simulations and the subsequent analysis make clear that this need not be the case. They show that the use of a binary encoding may lead to a phenomenon known as premature convergence. This phenomenon is an artifact that depends crucially on strategies being encoded in binary form.

Based on our findings, we conclude that in general the use of a binary encoding of strategies is undesirable. By not using a binary encoding, one avoids the risk of having to deal with all kinds of artifacts, such as the premature convergence observed by Alkemade et al. (2006, 2007, 2009). ${ }^{7}$ For various examples of studies in which EAs are employed without using a binary encoding, we refer to Sellgren (2001), Ünver (2001), Gerding et al. (2003), Lux and Schornstein (2005), Clemens and Riechmann (2006), Dawid and Dermietzel (2006), and Haruvy et al. (2006). It should be noted, however, that there are special cases in which we consider the use of a binary encoding perfectly acceptable. In a prisoner's dilemma, for example, agents have to make decisions that are intrinsically binary, namely decisions between cooperation and defection. The use of a binary encoding of strategies (e.g., Axelrod 1987) then seems a very natural choice that is unlikely to cause any artifacts.

The more general point that we want to make is that, when one employs an EA for economic modeling, all elements of the EA should have a meaningful economic interpretation (see also Dawid and Dermietzel 2006). ${ }^{8}$ Many EAs employed in economic research have been adopted from the computer science literature without any substantial modification. Such EAs are likely to contain elements of which the economic interpretation is unclear. The use of a binary encoding of strategies is an example of such an element. Other EA elements

\footnotetext{
${ }^{7}$ Our results seem to suggest that, if one insists on the use of a binary encoding of strategies, it is advisable to use a Gray coding rather than an ordinary binary encoding. However, even though in this paper we have not observed any artifacts of the use of a Gray coding, it seems quite well possible that such artifacts will be observed in other contexts. For example, the fact that in the case of a Gray coding the smallest and the largest decoded value differ by only one bit (see Table 2) seems unnatural and it may well be that this sometimes has unintended consequences.

${ }^{8}$ This point stands in stark contrast with one of the conclusions reached by Alkemade et al. (2006, 2007, 2009). They state that "economic model parameters and evolutionary algorithm parameters should be treated separately" (Alkemade et al. 2006, p. 367).
} 
of which the economic interpretation requires special attention include the population size (Alkemade et al. 2006, 2007, 2009), the selection operator (Van Bragt et al. 2001; Dawid and Dermietzel 2006), and the strategy updating regime (Dawid and Dermietzel 2006). As we have shown in this paper, EA elements without a sensible economic interpretation may lead to simulation results that lack a sound underlying economic rationale. To avoid such results, paying close attention to the economic interpretation of the various elements of an EA is absolutely essential.

Acknowledgement We thank an anonymous referee for various useful comments on an earlier draft of this paper.

Open Access This article is distributed under the terms of the Creative Commons Attribution Noncommercial License which permits any noncommercial use, distribution, and reproduction in any medium, provided the original author(s) and source are credited.

\section{References}

Alkemade F, La Poutré H, Amman HM (2006) Robust evolutionary algorithm design for socioeconomic simulation. Comput Econ 28(4):355-370

Alkemade F, La Poutré H, Amman HM (2007) On social learning and robust evolutionary algorithm design in the Cournot oligopoly game. Comput Intell 23(2):162-175

Alkemade F, La Poutré H, Amman HM (2009) Robust evolutionary algorithm design for socioeconomic simulation: a correction. Comput Econ 33(1):99-101

Andreoni J, Miller JH (1995) Auctions with artificial adaptive agents. Games Econom Behav 10(1):39-64

Arifovic J (1994) Genetic algorithm learning and the cobweb model. J Econ Dyn Control 18(1):328

Arifovic J (1996) The behavior of the exchange rate in the genetic algorithm and experimental economies. J Polit Econ 104(3):510-541

Arifovic J, Maschek MK (2006) Revisiting individual evolutionary learning in the cobweb model: an illustration of the virtual spite-effect. Comput Econ 28(4):333-354

Axelrod R (1987) The evolution of strategies in the iterated prisoner's dilemma. In Davis L (ed) Genetic algorithms and simulated annealing. Morgan Kaufmann, Los Altos, pp 32-41

Beyer H-G (2001) The theory of evolution strategies. Springer, Berlin

Beyer H-G, Schwefel H-P (2002) Evolution strategies: a comprehensive introduction. Nat Comput 1(1):3-52

Brenner T (2006) Agent learning representation: advice on modelling economic learning. In Tesfatsion L, Judd KL (eds) Handbook of computational economics, vol. 2. Elsevier, Amsterdam, pp 895-947

Bullard J, Duffy J (1998) A model of learning and emulation with artificial adaptive agents. J Econ Dyn Control 22(2):179-207

Casari M (2008) Markets in equilibrium with firms out of equilibrium: a simulation study. J Econ Behav Organ 65(2):261-276

Chattoe E (1998) Just how (un)realistic are evolutionary algorithms as representations of social processes? J Artif Soc Soc Simulat 1(3)

Clemens C, Riechmann T (2006) Evolutionary dynamics in public good games. Comput Econ 28(4):399-420

Dawid H (1996) Adaptive learning by genetic algorithms: analytical results and applications to economic models. Springer, Berlin

Dawid H, Dermietzel J (2006) How robust is the equal split norm? Responsive strategies, selection mechanisms and the need for economic interpretation of simulation parameters. Comput Econ 28(4):371-397 
Dawid H, Kopel M (1998) On economic applications of the genetic algorithm: a model of the cobweb type. J Evol Econ 8(3):297-315

Franke R (1998) Coevolution and stable adjustments in the cobweb model. J Evol Econ 8(4):383406

Gerding E, Van Bragt D, La Poutré H (2003) Multi-issue negotiation processes by evolutionary simulation, validation and social extensions. Comput Econ 22(1):39-63

Goldberg D (1989) Genetic algorithms in search, optimization, and machine learning. AddisonWesley, Reading

Hansen RG, Samuelson WF (1988) Evolution in economic games. J Econ Behav Organ 10(3):315338

Haruvy E, Roth AE, Ünver MU (2006) The dynamics of law clerk matching: an experimental and computational investigation of proposals for reform of the market. J Econ Dyn Control 30(3):457-486

Huberman BA, Glance NS (1993) Evolutionary games and computer simulations. Proc Natl Acad Sci 90(16):7716-7718

Lux T, Schornstein S (2005) Genetic learning as an explanation of stylized facts of foreign exchange markets. J Math Econ 41(1-2):169-196

Marks RE (1992) Breeding hybrid strategies: optimal behaviour for oligopolists. J Evol Econ 2(1):17-38

Maschek MK (2010) Intelligent mutation rate control in an economic application of genetic algorithms. Comput Econ 35(1):25-49

Miller JH (1986) A genetic model of adaptive economic behavior. Working paper, University of Michigan

Miller JH (1996) The coevolution of automata in the repeated prisoner's dilemma. J Econ Behav Organ 29(1):87-112

Mitchell M (1996) An introduction to genetic algorithms. MIT, Cambridge

Price TC (1997) Using co-evolutionary programming to simulate strategic behaviour in markets. J Evol Econ 7(3):219-254

Rhode P, Stegeman M (1996) A comment on "Learning, mutation, and long-run equilibria in games". Econometrica 64(2):443-449

Schaffer ME (1988) Evolutionarily stable strategies for a finite population and a variable contest size. J Theor Biol 132(4):469-478

Schaffer ME (1989) Are profit-maximisers the best survivors? A Darwinian model of economic natural selection. J Econ Behav Organ 12(1):29-45

Sellgren A (2001) The evolution of insurance markets under adverse selection. J Evol Econ 11(5):501-526

Ünver MU (2001) Backward unraveling over time: the evolution of strategic behavior in the entry level British medical labor markets. J Econ Dyn Control 25(6-7):1039-1080

Van Bragt D, Van Kemenade C, La Poutré H (2001) The influence of evolutionary selection schemes on the iterated prisoner's dilemma. Comput Econ 17(2-3):253-263

Vega-Redondo F (1997) The evolution of Walrasian behavior. Econometrica 65(2):375-384

Vriend NJ (2000) An illustration of the essential difference between individual and social learning, and its consequences for computational analyses. J Econ Dyn Control 24(1):1-19

Waltman L, Van Eck NJ (2009a) A mathematical analysis of the long-run behavior of genetic algorithms for social modeling. Working paper no ERS-2009-011-LIS, Erasmus University Rotterdam, Erasmus Research Institute of Management

Waltman L, Van Eck NJ (2009b) Robust evolutionary algorithm design for socio-economic simulation: some comments. Comput Econ 33(1):103-105

Wheeler S, Bean N, Gaffney J, Taylor P (2006) A Markov analysis of social learning and adaptation. J Evol Econ 16(3):299-319

$\mathrm{Xu}$ Y (2006) The behavior of the exchange rate in the genetic algorithm with agents having long memory. J Evol Econ 16(3):279-297 